\title{
La FMH à propos de la maîtrise des coûts de santé
}

\author{
Nora Willea, Jeanine Glarner ${ }^{b}$, Jürg Schlup ${ }^{c}$ \\ ${ }^{a}$ Dr phil., collaboratrice scientifique du président; ${ }^{b}$ Spécialiste en communication, division Communication de la FMH; ${ }^{c} \mathrm{Dr}$ méd., président de la FMH
}

\begin{abstract}
Garantir à l'avenir l'accès pour tous à une prise en charge médicale de qualité suppose une utilisation optimale des ressources. Cet article explore les potentiels d'efficacité inexploités du point de vue du corps médical.
\end{abstract}

\begin{abstract}
Les dépenses mensuelles d'un ménage moyen pour les primes d'assurance-maladie ont augmenté de 100 francs entre 2006 et 2014 . Même si cette hausse est inférieure à celles observées pour la charge fiscale (+370 CHF), la consommation (+325 CHF) ou l'épargne mensuelle $(+715 \mathrm{CHF})$ [1], les primes des caisses-maladie restent une charge notable pour les ménages. Pour faire face à l'évolution démographique et aux progrès de la médecine, qui exigeront des ressources croissantes, il est indispensable d'utiliser les moyens à disposition de la manière la plus efficace possible.

La responsabilité du corps médical ne se limite pas à chacun de ses patients, mais s'étend au système de
\end{abstract}

\section{Résumé}

Garantir l'accès pour tous à une prise en charge médicale de qualité suppose une utilisation optimale des ressources. Le corps médical estime qu'un énorme potentiel d'efficacité reste inutilisé, notamment au niveau des structures et du financement de notre système de santé. La création de régions hospitalières supra-cantonales, la suppression des conflits de gouvernance des cantons et une perspective davantage axée sur le bénéfice pour les patients dans la LAMal offrent ici des approches essentielles. Un financement uniforme des prestations ambulatoires et hospitalières est incontournable pour appliquer le principe de «l'ambulatoire avant l'hospitalier» sans hausse des primes et accroître l'attractivité des modèles de soins intégrés. Plusieurs mesures en faveur de la qualité sont également susceptibles de réduire les prestations de santé tout en améliorant la prise en charge médicale. La tarification et l'admission des médecins sont également des éléments capitaux, au même titre que la réduction des contraintes administratives. Toutes ces propositions seraient réalisables sans limiter la qualité des soins ni le libre choix du médecin. Dans ce contexte, toutes les mesures visant à restreindre le libre choix du médecin ou conduisant à un rationnement caché sont à bannir. santé de demain, qui doit garder sa qualité élevée, rester accessible à tous et financièrement supportable. Parce que les médecins, grâce à leur pratique quotidienne, connaissent parfaitement notre système de santé, les différents organes de la FMH ont élaboré des propositions visant à maîtriser les coûts. Nous constatons avec plaisir que le rapport du groupe d'experts [2] du DFI a repris une partie de ces propositions, même si de fortes divergences subsistent quant à leur application.

\section{Optimiser l'ensemble du système}

La Suisse s'offre 26 systèmes de santé pour une population de 8 millions d'habitants. La création de cinq à sept régions hospitalières supra-cantonales permettrait de réaliser des gains en efficacité et en qualité des soins tout en évitant des surcapacités. Il faut aussi revoir les prérogatives des cantons; à la fois planificateurs et propriétaires d'hôpitaux, demandeurs ou financeurs de prestations hospitalières, mais aussi régulateurs des listes hospitalières et des tarifs, ce qui conduit à une inefficacité et à des distorsions de concurrence. Les cantons devraient au moins renoncer à la propriété d'hôpitaux.

Un autre potentiel d'amélioration pourrait être réalisé en s'éloignant d'une perspective purement axée sur les coûts de l'assurance-maladie (LAMal) vers une perspective plus globale, prenant aussi en compte les bénéfices des traitements pour les patients. Si, par analogie à la loi sur l'assurance-accidents (LAA), la LAMal prenait en compte non seulement les coûts de traitement (qui, en comparaison, restent raisonnables), mais aussi les coûts indirects liés aux arrêts de travail et aux rentes, les investissements dans des traitements performants seraient intéressants non seulement pour les patients, mais aussi pour les assureurs et auraient, à la clé, un impact positif sur l'économie. 


\section{Réaliser un potentiel d'économie par un financement uniforme}

Le seul passage à un financement uniforme des prestations ambulatoires et hospitalières permettrait d'exploiter le plus important potentiel d'efficacité du système de santé, car le transfert des prestations hospitalières vers le domaine ambulatoire offre des possibilités d'économies annuelles d'un milliard de francs [3]. Remplacer des traitements hospitaliers financés surtout par l'impôt par des traitements ambulatoires exclusivement financés par les primes exige un financement uniforme de l'ensemble de ces prestations si l'on veut éviter une hausse des primes. Une telle approche permettrait aussi de mieux exploiter le potentiel des soins intégrés. Principalement dans le domaine des maladies non transmissibles, à l'origine de $80 \%$ des coûts de santé [4], la participation volontaire à des modèles de soins intégrés a généré d'importantes économies [5]. La répercussion insuffisante des économies réalisées grâce aux hospitalisations évitées rend aujourd'hui ces modèles peu attrayants, que ce soit pour les assurés ou pour les caisses-maladie. Actuellement, une caisse-maladie qui favorise les traitements ambulatoires meilleur marché voit ses primes augmenter. Par conséquent, un potentiel d'efficacité s'élevant jusqu'à deux milliards de francs est négligé chaque année [6].

\section{Investir dans la qualité}

La qualité dans le domaine de la santé est une tâche principale du corps médical et joue un rôle essentiel à la fois dans le quotidien médical et dans la politique professionnelle. La démarche qualité repose sur trois piliers, la transparence, la responsabilité et la durabilité [7]. Des initiatives comme smarter medicine montrent qu'il est possible d'allier qualité et économies. Prescrire moins peut être le mieux pour un patient et lui épargner des souffrances inutiles [8]. Le médecin doit pour cela prendre ses décisions en partenariat avec le patient et sur la base de données étayées. Une augmentation des compétences en santé de la population aura un effet modérateur sur les coûts, par ex. à l'aide d'informations sur l'intérêt d'attendre ou la pondération risques-bénéfices. Lorsque le choix d'un traitement n'est pas évident, les patients peuvent déjà aujourd'hui recourir à un second avis médical pour assurer une indication - une démarche soutenue par les membres de la FMH (art. 16 du Code de déontologie). Contraindre légalement à un second avis médical reviendrait cependant à mettre les patients sous tutelle. Le recours à plus d'expertise au sein de conseils d'indication (boards) a fait ses preuves, et les professionnels impliqués ou leur société de disci- pline médicale sont les mieux placés pour juger au cas par cas de leur nécessité.

Comme l'efficacité, l'adéquation et le caractère économique d'une prestation médicale, et donc l'obligation de remboursement de l'AOS, ne peuvent être appréciés que sur la base d'un examen scientifique transparent, il faut des bases de décision consolidées à l'image des Health Technology Assessments (HTA). De la même manière, les guidelines reprennent les connaissances actuelles et permettent d'agir simultanément sur les coûts et la qualité des soins. Ils doivent cependant laisser suffisamment de latitude pour tenir compte des spécificités des patients polymorbides. Pour répondre au besoin de données validées, la FMH défend la promotion des registres médicaux [9].

Toutes les mesures de qualité doivent être appréciées en fonction du surcroît de travail administratif et de la nécessaire liberté thérapeutique: l'exercice de la médecine dans les règles de l'art ne doit entraîner aucun préjudice, ni pour les médecins ni pour les patients. Ceci vaut également pour les contrats de médecins hospitaliers proposant des bonus liés à des indicateurs purement économiques, comme des objectifs quantitatifs ou un chiffre d'affaires. Les hôpitaux proposant des bonus liés à des objectifs quantitatifs devraient être exclus des listes hospitalières cantonales.

\section{Renforcer les soins ambulatoires par une juste tarification}

La tarification présente, elle aussi, un potentiel d'optimisation, par ex. pour les prestations identiques entre les secteurs ambulatoire et hospitalier, mais rémunérées différemment. L'application systématique du principe de «l'ambulatoire avant l'hospitalier» exige un renforcement des soins ambulatoires. C'est pourquoi un élément central de la révision du TARMED consiste à rendre à la structure tarifaire son caractère approprié et conforme aux règles applicables en économie d'entreprise, base nécessaire à des forfaits dans le domaine ambulatoire. Comme le partenariat tarifaire est le meilleur moyen d'atteindre cet objectif, le corps médical contribuera lui aussi au succès de la révision tarifaire. Il pourrait être utile de créer un bureau tarifaire national analogue à SwissDRG qui serait chargé du suivi du tarif, pour autant que l'autonomie de la structure et de l'organisation soit garantie et que le Conseil fédéral se limite comme aujourd'hui à approuver le tarif proposé.

\section{Réduire l'administration}

Le potentiel d'efficacité d'une réduction des charges administratives est une évidence pour les médecins en 
Suisse: la gestion des dossiers médicaux prend, à elle seule, $19 \%$ du temps de travail des médecins hospitaliers et a augmenté de 26 minutes par médecin et par jour depuis 2011 [10]. Rien que ce temps supplémentaire correspond à 662 postes de médecins (à 56 heures hebdomadaires). Cela devrait donner à réfléchir au manque de relève et de réglementation pour les admissions, car la charge administrative augmente aussi dans le domaine ambulatoire: la part des médecins de premier recours qui consacrent plus à la $75 \%$ de leur temps au contact avec les patients a diminué de 60 à $45 \%$ entre 2012 et 2015 [11]. L'administration absorbe du temps de travail et génère des coûts. Pourtant, cet immense potentiel d'économies ne suscite guère l'attention politique.

\section{Piloter l'admission par des critères de qualité}

La politique mise sur une régulation du nombre de praticiens alors que même du côté des caisses-maladie, on souligne qu'il «n'existe pas de corrélation entre la densité de médecins et les prestations médicales aux patients» et que la densité médicale «n'est toutefois pas un outil adéquat de contrôle des volumes» [1]. Le fait que le nombre de postes de médecins vacants a plus que doublé ces quatre dernières années [12] et que la stratégie de «l'ambulatoire avant l'hospitalier» exige un développement du domaine ambulatoire fait penser que les approches discutées pour limiter l'installation de médecins en cabinet risquent d'être peu concluantes.

C'est pourquoi la FMH continue de soutenir des critères de qualité cumulatifs comme condition d'admission, alliant la qualité des soins à un pilotage économique efficace avec, premièrement, l'exercice d'une activité médicale pendant trois ans dans un établissement de formation postgraduée reconnu dans la discipline demandée pour l'admission, deuxièmement, une attestation de formation continue et, troisièmement, la justification des compétences linguistiques dans la langue officielle de la région d'activité. La gestion de l'admission en vigueur depuis 2013 a déjà réduit les ouvertures de cabinets de $33 \%$. La réglementation plus stricte pro- posée par la FMH permettrait un choix plus efficace selon des exigences de qualité transparentes, sans toucher au libre choix du patient. En revanche, d'autres propositions comme la suppression de l'obligation de contracter signent la fin du libre choix du médecin, accordent trop de poids aux objectifs économiques des assureurs-maladie et pénalisent les patients.

\section{Conclusion}

Les propositions de solutions évoquées ici montrent qu'un énorme potentiel d'efficacité reste actuellement inutilisé, notamment au niveau des structures et du financement de notre système de santé; ce potentiel pourrait être exploité sans porter préjudice à la qualité des soins ni au libre choix du patient. Dans une telle situation, les mesures limitant le libre choix du médecin ou conduisant à un rationnement caché sont à bannir.

\section{Références}

1 Früh M, Gyger P, Reich O. Rapport d'Helsana «Evolution des dépenses en matière de santé». Décembre 2016.

2 Rapport du groupe d'experts. "Mesures visant à freiner la hausse des coûts dans l'assurance obligatoire des soins». 24 août 2017.

3 Pwc. «Ambulatoire avant hospitalier. Ou comment économiser un milliard de francs chaque année». Août 2016.

4 OFSP. Une étude présente pour la première fois le calcul des coûts directs et indirects des principales maladies non transmissibles. Bulletin. 2014;36(14):583-6.

5 Huber CA, Reich O, Früh M, Rosemann T. Effects of Integrated Care on Disease-Related Hospitalisation and Healthcare Costs in Patients with Diabetes, Cardiovascular Diseases and Respiratory Illnesses: A Propensity-Matched Cohort Study. International Journal of Integrated Care. 2016;16(1):1-18.

6 Klaus G. Plus grande efficacité grâce au financement uniforme. Point de vue Helsana. 2016;4:3-5.

7 Bosshard C. Charte qualité: transparence, responsabilité et durabilité. Bull Méd Suisses. 2016;97(45):1549.

8 La position de la FMH. «Choosing wisely»: moins de prestations pour davantage d'utilité. Approuvée par le Comité central de la FMH. Berne, 15 décembre 2016.

9 Position de la FMH. Les registres médicaux: un outil majeur d'assurance-qualité en médecine. Berne, août 2011.

10 Golder L, et al. Environnement de travail en mutation et attitude envers de nouveaux modèles de financement. Effets visibles de l'orientation vers la performance dans le domaine de santé. Rapport final de gfs.bern sur l'étude concomitante sur mandat de la FMH. Janvier 2018.

11 OBSAN. Analyse de l'International Health Policy Survey (IHP). 2012 et 2015 .

12 Schweizer Jobradar. Rapport de postes vacants. $4^{\mathrm{e}}$ trimestre $2017 \mathrm{et}$ $4^{\mathrm{e}}$ trimestre 2013 en comparaison. URL: https://www.jobagent.ch/ jobradar 